\title{
AN ELECTRICAL CONDUCTIVITY TRANSLATOR FOR CARBONS
}

\author{
N. Rey-Raap, E.G. Calvo, J.M. Bermúdez, I. Cameán, A.B. García, J.A. Menéndez, A. \\ Arenillas* \\ Instituto Nacional del Carbón INCAR-CSIC, Apartado 73, 33080 Oviedo, Spain
}

\begin{abstract}
The variety of methodologies used to determine the electrical conductivity of carbons makes it very difficult to compare samples and establish reference values. In this study, the electrical conductivity of a wide range of carbons was determined using two different methods: fourpoint probe and compression. Although the methodologies and the operating conditions are very different, linear correlations between the values measured by these two methods can be established for some of the materials studied. Only materials with a very high conductivity (graphite and carbon black) could not be correlated.
\end{abstract}

\section{Keywords}

amorphous materials; microporous materials; nanostructures; electrical conductivity

\section{Introduction}

Electrical conductivity (i.e. the ability of a material to conduct electric current), is a very important property, especially in materials used for energy storage [1,2], metal-polymer composites manufacture [3], electronic devices, etc. For this reason, it is essential to employ a simple and reliable method to determine the electrical conductivity as a control property of many solid materials after their production. However, as yet, there is no worldwide accepted method for carrying out such control, as there is for other properties. The disparities between the methodologies employed to determine electrical conductivity (i.e. impedance spectroscopy [4], the Van der Pauw method [5], the measurement of conductivity by compression [6]) make it necessary to find values that can be used to compare different materials. Although all of these methods provide electrical conductivity data, the procedure,

\footnotetext{
*Corresponding autor: E-mail: aapuente@incar.csic.es

Phone: +34985119090

Apartado 73, 33080 Oviedo SPAIN
} 
the measuring device, the operating conditions, etc. are different, and so the so-called "conductivity" value of the material needs to be treated differently in each case.

In this study, the electrical conductivity of various carbonaceous materials was determined using the two most common methods that can be found in the literature, four-point probe technique and measure under compression [5-8], with the aim of finding out whether they can be correlated. The first method consists in preparing disc-shaped pellets and measuring their sheet resistivity by means of the four-point probe (FPP) technique (based on the Van der Pauw equation), whereas the second involves monitoring the electrical conductivity of powdery samples under compression (COM) in a specific pressure range. The results are compared and various aspects related to the operating conditions are evaluated taking into account the advantages and disadvantages of each method.

\section{Materials and Methods}

The following commercial carbons were selected for this research: 7 activated carbons applied in electrochemical systems (Supra 30, Super 30 and Supra 50 from NORIT, YEC-8A and YEC-8B from Fuzhou Yihuan Carbon, SO-15A from TDA Research and YP-50F from Kuraray); 2 generic carbon xerogels (XER-HMV and XER-HSA supplied by Xerolutions); carbon fibers (AS4C-3K from Hexcel Core); a graphite (TIMREX SLP50) and a carbon black (Super P-Li from Timcal). Some in-lab samples obtained from anthracites by different treatments $[9,10]$ were also used.

FPP measurements were performed by applying a four-point contact at the edge of the conducting surface (model SR-4-6L, Everbeing). Once the probes were in contact with the surface of the material, a constant current (in the range of 9-10 mA) was applied through the two outer tungsten pins (DC current source model 6220, Keithley) and the corresponding voltage drop was measured across the two inner tungsten pins (digital nanovoltmeter model 2182A, Keithley). All the materials tested were thin pellets (diameter: $10 \mathrm{~mm}$; weight: 13-15 mg; thickness: 200-500 $\mu \mathrm{m}$ ) formed from a mixture of the active material (90 wt. \%) and a binder, PTFE, (10 wt. \%). The mixture was rolled out and punched and the pellets obtained were subjected to a five-ton pressure for 10 seconds before the measurements of sheet resistivity at ambient conditions (i.e. room temperature and atmospheric pressure) were performed. Pellets of carbon black without any binder were also prepared to evaluate the effect of the binder on the measured value. This procedure is in accordance with the general principles of the ASTM standard methods D257-99 and D4496-87.

For the determination of conductivity by means of compression (COM), the sample was 
introduced into a polyethylene cylinder with a cross-section of $0.44 \mathrm{~cm}^{2}$ into whose ends were placed two bronze pistons connected to a source of electric current (Time electronics 1044) and to a potentiometer (Fluke 45 Dual display multimeter). The height of the samples inside the cylinder was measured with a precision laser device (Keyence LK-2101). An external pressure was applied to the pistons in order to compress the sample and hence reduce its height. Firstly, the optimum amount of each material needed to obtain the maximum electrical conductivity was determined by representing the conductivity value versus the corresponding height of the sample column measured for each pressure in the range of 0.01 MPa-7.00 $\mathrm{MPa}$. In this work, the amount of carbon samples used ranged between 100 and $800 \mathrm{mg}$. The optimised amount of material was introduced into the cylinder and compressed by the pistons up to $150 \mathrm{MPa}$, using a hydraulic press until a constant potential difference was obtained. The electrical conductivity was calculated according to $\sigma=\mathrm{I} \cdot \mathrm{h} / \phi \cdot \mathrm{A}$, where $\sigma$ is the electrical conductivity $\left(\mathrm{S} \mathrm{cm}^{-1}\right) ; I$ is the current intensity $(\mathrm{mA}), h$ is the height of the column sample (cm), $\phi$ is the potential difference $(\mathrm{mV})$ and $A$ is the cross-section of the cylinder $\left(\mathrm{cm}^{2}\right)$. All the measurements were performed using the polyethylene $0.44 \mathrm{~cm}^{2}$ cylinder (diameter 7.5 $\mathrm{mm}$ ) at a current density of $20 \mathrm{~mA}$. The height of the samples ranged between 0.5 and $3.0 \mathrm{~cm}$ depending on the type of carbon material used.

\section{Results}

The electrical conductivities of the materials measured by FPP and COM are presented in Table 1. As can be seen, the values determined by these two methods are very different in all cases. The COM method provides values that are much higher than the corresponding data obtained by the FPP method, except for the graphite. The reason for these higher conductivity measurements is that the COM method determines the electrical conductivity through a column of sample, whilst the FPP method measures the electrical conductivity transmitted along the surface of the material. It is essentially this difference that gives rise to the many other differences between these two methods, not only in the operating conditions (sample mass, pressure, etc.) but also a series of intrinsic properties of the sample that can influence differently on the electrical conductivity values measured from these two methods. Therefore, differences in the electrical conductivity of single particles, but also their size and morphology, the packing and contacts between particles, etc. can influence in a different way in each methodology. Moreover, the anisotropy of the sample, possible inhomogeneities or impurities, and the orientation of the more ordered structures within the sample to be measured, play also relevant roles in the resultant conductivity value. 
Table 1. Electrical conductivity of a series of carbons measured by two methods: four-point probing (FPP) and compression (COM), and the estimated FPP values from COM-FPP correlation

\begin{tabular}{|c|c|c|c|}
\hline Carbons & $\begin{array}{c}\text { FPP } \\
\left(\mathrm{S} \mathrm{cm}^{-1}\right)\end{array}$ & $\begin{array}{c}\text { COM } \\
\left(\mathrm{S} \mathrm{cm}^{-1}\right)\end{array}$ & $\begin{array}{c}\text { Estimated FPP } \\
\qquad\left(\mathrm{S} \mathrm{cm}^{-1}\right)\end{array}$ \\
\hline \multicolumn{4}{|l|}{ Activated carbons } \\
\hline Supra 30 & 0.59 & 19.01 & 0.53 \\
\hline Super 30 & 0.61 & 19.21 & 0.53 \\
\hline Supra 50 & 0.55 & 20.60 & 0.57 \\
\hline YEC-8A & 0.31 & 7.06 & 0.24 \\
\hline YEC-8B & 0.33 & 14.75 & 0.43 \\
\hline SO-15A & 0.42 & 14.67 & 0.42 \\
\hline YP-50F & 0.32 & 13.22 & 0.39 \\
\hline \multicolumn{4}{|l|}{ Carbon xerogels } \\
\hline XER-HMV & 0.13 & 0.88 & 0.09 \\
\hline XER-HSA & 0.35 & 12.98 & 0.38 \\
\hline \multicolumn{4}{|c|}{ Carbonized anthracites } \\
\hline AF20C900 [9] & 0.16 & 4.40 & 0.18 \\
\hline ATO20C1000 [9] & 0.28 & 9.70 & 0.30 \\
\hline \multicolumn{4}{|l|}{ Carbon fibers } \\
\hline AS4C-3K & 0.71 & 25.85 & 0.69 \\
\hline \multicolumn{4}{|l|}{ Graphite } \\
\hline Timrex SLP50 & 1831 & 50.23 & n.a. \\
\hline \multicolumn{4}{|l|}{ Carbon black } \\
\hline Super P-Li & 9.30 & 64.26 & n.a. \\
\hline \multicolumn{4}{|c|}{ Graphitized materials } \\
\hline $\mathrm{AF} / 2600[9]$ & 5.58 & 29.90 & 4.88 \\
\hline B/CIQ1/2000 [10] & 11.72 & 37.00 & 10.23 \\
\hline B/CIQ1/2300 [10] & 17.51 & 48.70 & 19.05 \\
\hline $\mathrm{A} / \mathrm{CVP} / 2600[10]$ & 27.22 & 58.90 & 26.74 \\
\hline
\end{tabular}




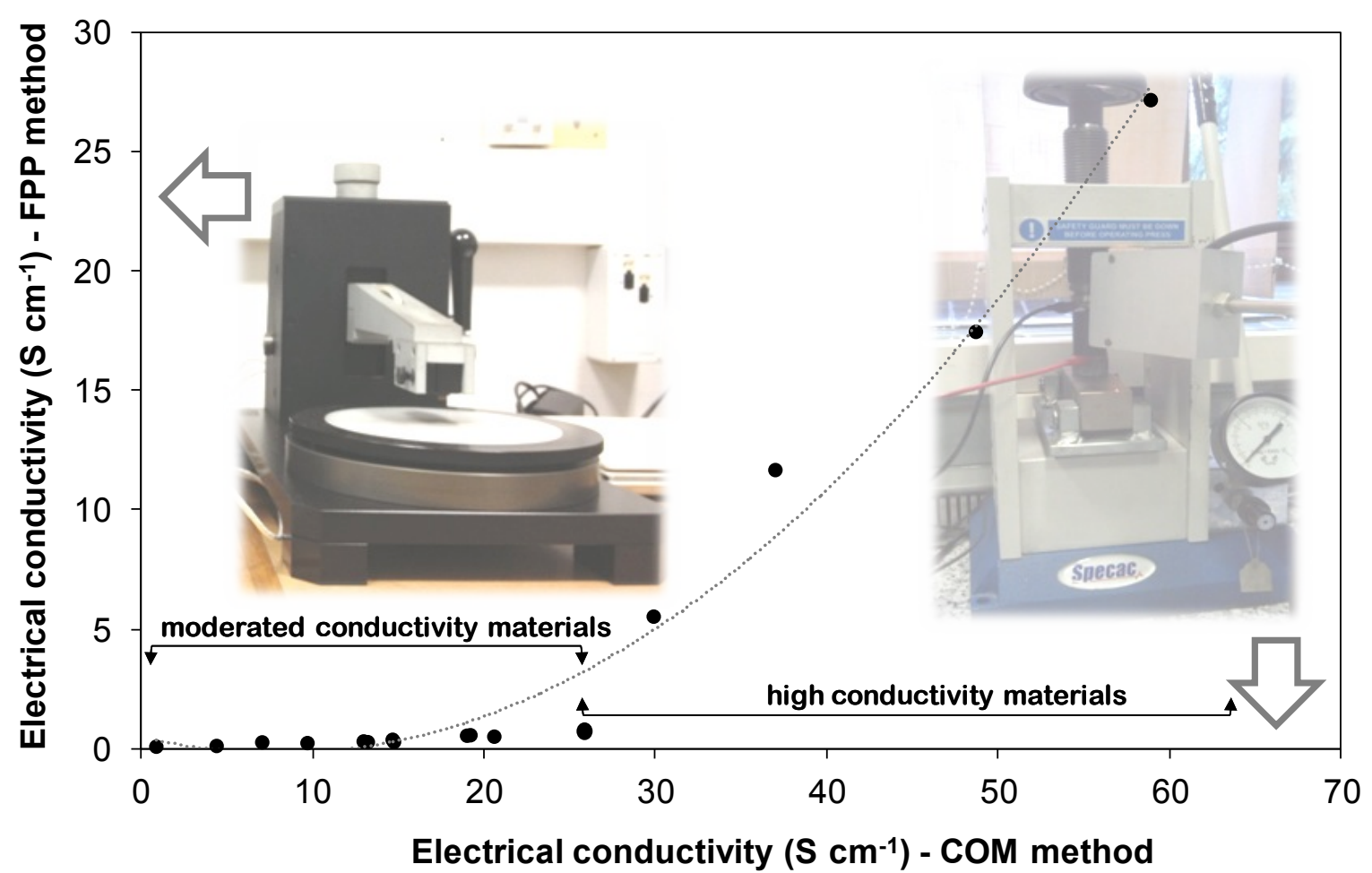

Figure 1. Relationship between the electrical conductivity measured by four-point probe (FPP) and compression (COM) for the series of materials studied.

If the conductivity values (excluding graphite and carbon black) reported in this work are compared, two very well defined categories emerge (see Figure 1): (i) materials with a moderate COM conductivity (up to $25 \mathrm{~S} \mathrm{~cm}^{-1}$ ) and very low FPP values, and (ii) materials with a high COM conductivity $\left(25-60 \mathrm{~S} \mathrm{~cm}^{-1}\right)$ and FPP values of the same order of magnitude. The inflexion point appears to be located at around $25 \mathrm{~S} \mathrm{~cm}^{-1}$ for COM conductivity (i.e. value of the carbon fibers, Table 1). From the results obtained, it is clear that there is no inconsistency between the two methods, i.e. the samples with higher conductivity present higher values in both cases. This suggests that it might be possible to correlate the two methods.

To explore the possibility of establishing correlations between the electrical conductivity determined by the FPP and COM methods, linear regression analysis was performed using the data corresponding to the materials of moderate COM conductivity (Figure 2) as well as the data from the in lab-graphitized materials (AF/2600, BCIQ1/2000, BCIQ1/2300, A/CVP/2600 in Table 1) that exhibit a high COM conductivity (Figure 3). 


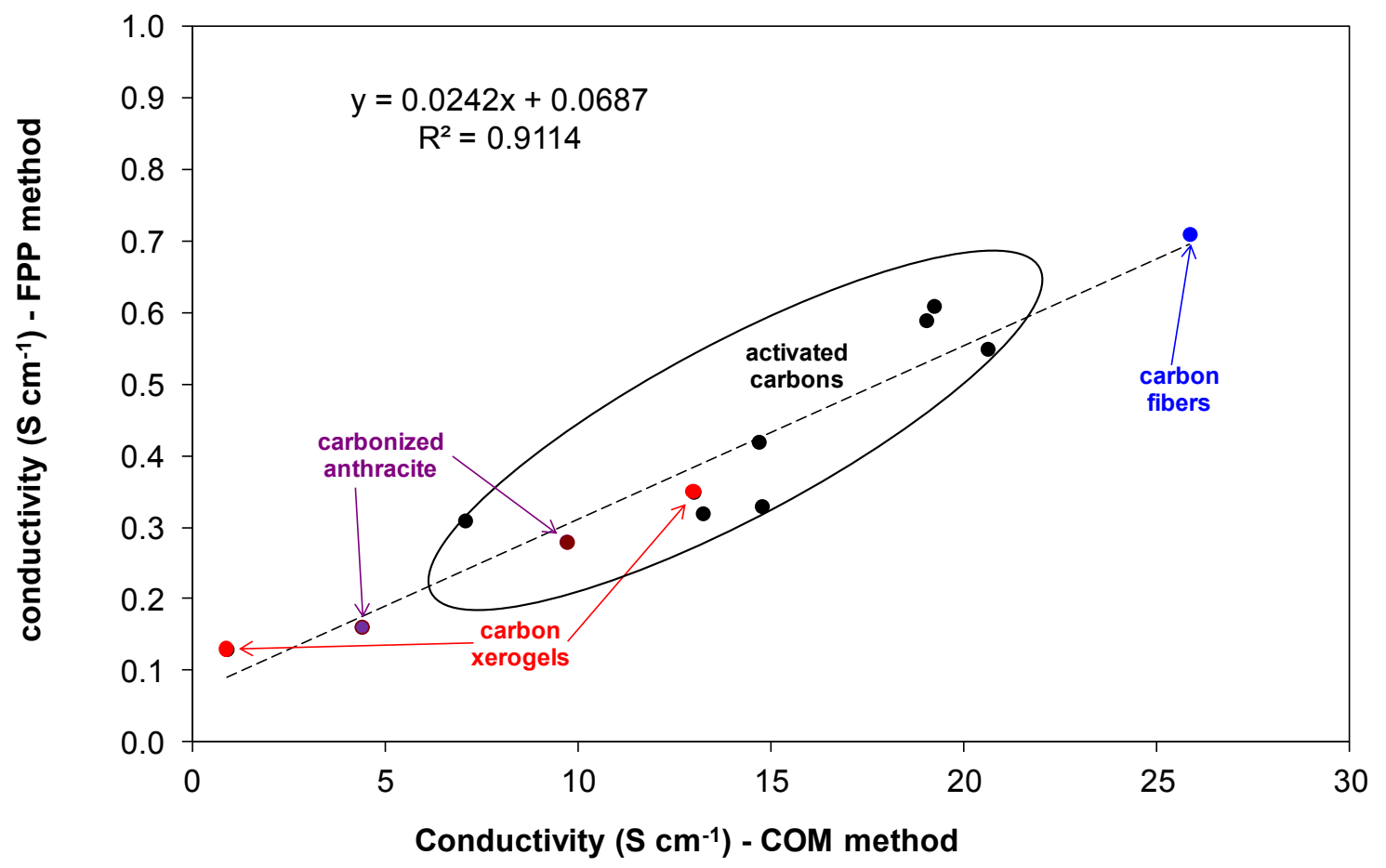

Figure 2. Correlation between the electrical conductivity measured by four-point probing (FPP) and compression (COM) for the materials with moderate conductivity.

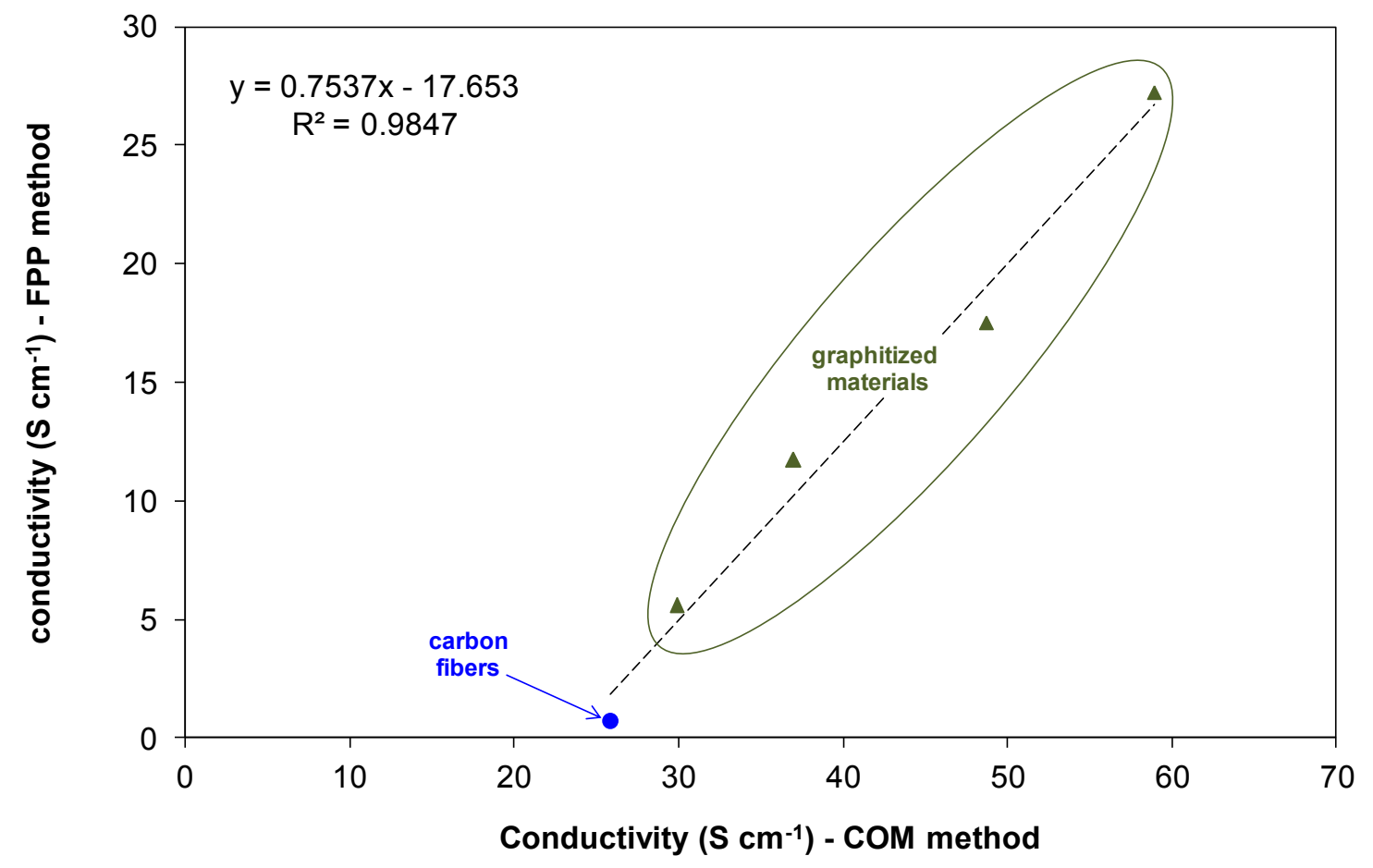

Figure 3. Correlation between the electrical conductivity measured by four-point probing (FPP) and compression (COM) for the materials with a high conductivity. 
As can be seen, good linear relationships were established between both types of materials. By using these correlations, the FPP electrical conductivities of the materials were calculated (Estimated FPP values in Table 1). The estimated and the experimentally measured FPP values are basically similar, thus confirming the validity of the correlation between COM and FPP methods. Unlike the graphitized materials, the graphite and the carbon black tested which also have high COM electrical conductivities do not fit any correlation. Furthermore, the measured FPP conductivity of graphite is much higher than that obtained with the COM method (1831 S cm${ }^{-1}$ vs. $\left.50.23 \mathrm{~S} \mathrm{~cm}^{-1}\right)$. This apparently anomalous result can only be explained on the basis of the anisotropic nature of graphite which leads to an overestimation of the FPP measurement of electrical conductivity. This type of structural ordered carbons is a very good conductive material in the in-plane (graphene plane) direction. There is no doubt therefore, that the degree of anisotropy of these materials has a pronounced influence on the value of the FPP measurements. On the basis of the differences between the FPP and COM values, the materials studied can be classified into two groups: (i) non structurally ordered or non-anisotropic materials (activated carbons, carbon xerogels, carbonized anthracites, carbon fibers, carbon black) with relatively high COM/FPP ratios $(>6)$ and (ii) structurally ordered materials with different degrees of anisotropy, such as the graphite and the graphitized materials prepared in our laboratory that have low COM/FPP ratios $(<6)$. As mentioned above, the determination of conductivity by means of the four-point probe method involves the use of pellets which are composed of the active material and a binder $(10 \% \mathrm{wt}$. of PTFE was used as binder in this study). For this reason, it would be useful to know whether the presence of this additive influences the measurements and gives a distorted value of electrical conductivity. The possible interference of the binder on the FPP method was evaluated using the carbon black, Super P-Li, as active material. The electrical conductivity was determined both with and without the binder and it was found that both values were very similar (i.e. 9.30 $\mathrm{S} \mathrm{cm}^{-1}$ in the case of the pellet composed of $100 \% \mathrm{wt}$. of carbon black versus $8.93 \mathrm{~S} \mathrm{~cm}^{-1}$ when $10 \%$ wt. of PTFE was used to prepare the pellet). In order to corroborate this result, the potential influence of the binder was also evaluated by measuring the electrical conductivity with the COM method. The electrical conductivity was measured with the same pure carbon black and also with a mixture of this carbon black with the polymer binder. The result was the same than that obtained with the other methodology since there was no great difference between the two values (i.e. 64.26 and $62.17 \mathrm{~S} \mathrm{~cm}^{-1}$ with and without the binder). It seems clear, therefore, that a relation can be established between the two methods, FPP and COM, 
even in the case of the FPP method where a binder is necessary for the preparation of the pellets.

\section{Conclusions}

The results discussed have highlighted some of the advantages of each method. Thus, FPP has the great advantage that it is very rapid and simple to perform under ambient conditions, and only a small amount of sample is needed to evaluate the electrical conductivity of the carbon materials, while the COM method is particularly useful for either non-compact or graphitic materials with a high degree of anisotropy.

In summary, it has been demonstrated that by applying the correlations defined in this work, it is possible to transfer the electrical conductivity values of several types of carbonaceous materials measured by the FPP and COM methods, thereby making it possible to compare the different data reported in the literature.

\section{Acknowledgements}

Financial support from the Ministerio de Economía y Competitividad of Spain MINECO (under Projects MAT2011-23733, IPT-2012-0689-420000 and ENE2011-28318) is greatly acknowledged. NRR is also grateful to MINECO for her predoctoral research grant.

\section{References}

[1] Dangles C. Rane-Fodacarao M. Devarajan TS. Higashiya S. Snyder J. Haldar P. Role of conducting carbon in electrodes for electric double layer capacitors. Materials Letters 2011; 65:300-303.

[2] Spahr ME. Goers D. Leone A. Stallone S. Grivei E. Development of carbon conductive additives for advanced lithium ion batteries. Journal of Power Sources 2011; 196:3404-3413. [3] Psarras GC. High conductivity in polymer matrix-metal particles composites. Composites Part A: Applied Science and Manufacturing 2006; 37:1545-1553.

[4] Mikhailenko SD. Guiver MD. Kaliaguine S. Measurements of PEM conductivity by impedance spectroscopy. Solid State Ionics 2008; 179: 619-624.

[5] Van der Pauw LJ. A method of measuring specific resistivity and Hall effect of discs of arbitrary shape. Philips Research Reports 1958; 13: 1-9.

[6] Marinho B. Ghislandi M. Tkalya E. Konning CE. de With G. Electrical conductivity of compacts of grapheme, multi-walled carbon nanotubes, carbon black and graphite powder. Powder Technology 2012; 221: 351-358. 
[7] A. Celzard, J.F. Mareche, F. Payot, G. Furdin. Electrical conductivity of carbonaceous powders. Carbon 40, 2801-2815, 2002.

[8] X. Shui, D.D.L. Chung. Electrical resistivity of submicron-diameter carbon-filament. Carbon 39, 1717-1722, 2001.

[9] D. González, M. A. Montes-Morán, I. Suárez-Ruiz, A. B. Garcia. Structural characterization of graphite materials prepared from anthracites of different characteristics: a comparative analysis. Energy \& Fuels 18, 365-370, 2004.

[10] M. Cabielles, M. A. Montes-Morán, A. B. Garcia. Structural study of graphite materials prepared by HTT of unburned carbon concentrates from coal combustion fly ashes. Energy \& Fuels 22, 1239-1243, 2008. 


\section{HIGHLIGHTS}

- Four points probe is adequate for small amount of carbon samples

- Compression method is preferable for anisotropic carbons

- Carbon conductivities from both methods can be correlated allowing comparison 